\title{
Société vaudoise de médecine
}

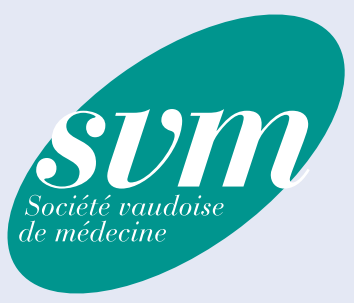

Fondée en 1829, la Société vaudoise de médecine compte 2500 membres, ce qui en fait la $3^{\mathrm{e}}$ de Suisse par la taille, actifs dans tous les domaines de la médecine. La SVM est organisée en groupements dont le dernier né est lié à l'évolution prévisible du managed care.
Société vaudoise de médecine

Président:

Charles-Abram Favrod-Coune

Secrétaire général:

Pierre-André Repond
Correspondance:

Société vaudoise de médecine

Chemin de Mornex 38

Case postale 7443

$\mathrm{CH}-1002$ Lausanne

Tél. 0216510505

Fax 0216510500

info@svmed.ch

www.svmed.ch
Toutes les spécialités, les régions et les domaines spécifiques sont représentés au sein d'une assemblée des délégués, parlement des médecins vaudois, grâce auquel des positions fortes se sont dégagées malgré les turbulences de ces dernières années.

\section{Les particularités de la SVM}

- Elle défend les intérêts des médecins installés comme ceux des médecins hospitaliers.

- Elle a choisi de développer ses propres outils informatiques en créant son Centre de confiance. Elle a pu ainsi élaborer un outil de facturation pour les médecins.

- Elle assume plusieurs mandats dont un mandat de gestion de la garde médicale et le secrétariat de la Société Médicale de la Suisse Romande.

La forte identité de la SVM ne l'empêche nullement de participer pleinement à la politique professionnelle au plan régional et fédéral en s'impliquant autant que possible dans les instances concernées: FMH, Conférence des sociétés cantonales de médecine, NewIndex, Société Médicale de la Suisse Romande, etc. Elle est ouverte à toute forme de coopération avec d'autres sociétés cantonales ou de spécialistes, indispensables dans le contexte actuel.

\section{Les principaux métiers de la SVM}

- L'action politique: la campagne intense menée par les médecins vaudois lors de la récente votation et le rejet historique à près de $90 \%$ de l'article 117 a témoigne des progrès accomplis.

- La négociation: l'aboutissement des difficiles négociations avec santésuisse a permis de conclure une convention incluant le règlement de la compensation TARMED (24 mil- lions de francs) en faveur des médecins sous forme d'un complément à la valeur de point, portée ainsi à Fr. -.99, tout en alimentant un fonds en faveur des autres cantons.

- A relever dans le secteur hospitalier la négociation et le suivi d'une $1^{\text {re }}$ convention collective de travail des médecins-chefs.

- La communication à travers notamment le Courrier du médecin vaudois et notre site www.svmed.ch mais aussi les relations avec les media et diverses publications.

- La médiation de conflits entre médecins et patients ou entre médecins.

- Les services aux membres: conseils juridiques individuels ou collectifs (plateforme de remise de cabinets, installation, retraite, gestion du personnel, assurances, action contre okdoc, etc.), contrats-cadre préférentiels (RC, assurances complémentaires, LPP, etc.).

- La formation continue: les Jeudis de la vaudoise et la médecine d'urgence.

- L'analyse et la gestion de projets: il faut une vigilance constante pour anticiper les évolutions, combattre ce qui doit l'être ou s'adapter, en diligentant au besoin les études nécessaires.

\section{Les instruments}

La SVM peut compter sur un comité soudé travaillant en harmonie avec les autres organes et le secrétariat général. Des efforts accrus doivent être encore entrepris pour créer une parfaite cohésion avec tous nos membres.

\section{L'avenir}

Dans Société vaudoise de médecine il y a le mot médecine, c'est-à-dire le lieu de rencontre du médecin avec son patient. C'est donc à la liaison du corps médical avec les patients que notre société doit s'engager à l'avenir. 